\title{
Grenzen der rationalen Abschreckung: Psychologische Korrelate von aggressivem Verhalten in experimentellen Krisenverhandlungsspielen
}

\author{
Hanja Blendin/Gerald Schneider
}

Eine erste Version dieses Artikels wurde an der PVS-Autorenkonferenz an der Universität Mainz, 25.-27. September 2014 präsentiert. Wir danken dem Publikum, unserem Diskutanten Boris Egloff sowie den Herausgebern des Sonderheftes für hilfreiche Kommentare. Dieser Artikel entstand dank finanzieller Unterstützung für die Forschungsinitiative „Science of Social Stress and Conflict Resolution“ (SoSSCR) im Rahmen der 3. Förderlinie der Exzellenzinitiave. Urs Fischbacher, Kate Bendrick und Irenäus Wolff vom Thurgauer Wirtschaftsinstitut (TWI) haben uns bei der Programmierung wertvolle Unterstützung gewährt. Replikationsmaterial und weitere Angaben zum hier vorgestellten Experiment sind auf der Replikations-Homepage des zweiten Autors zu finden: http://www.polver. uni-konstanz.de/gschneider/arbeitspapiere/replikationsdaten/

\section{Einleitung}

Die Weiterverbreitung von Atomwaffen und die Gefahr des Erwerbs von Massenvernichtungswaffen durch terroristische Organisationen haben die Debatte über die Wirksamkeit der Abschreckung erneuert. Auf der einen Seite schreiben die Befürworter der Theorie rationaler Abschreckung diesem sicherheitspolitischen Instrument einen deeskalierenden Einfluss zu. Dazu gehört nicht zuletzt der Nobelpreisträger Thomas Schelling (2006, S. 6093), der Ende der 1950er und Anfang der 1960er Jahre den Aspekt der Glaubwürdigkeit als zentrales Abschreckungsmittel identifizierte und der in seiner Vorlesung zur Überreichung des Nobelpreises für Wirtschaftswissenschaften auf die Bedeutung der spieltheoretisch fundierten Abschreckungstheorie für die Sicherheitspolitik verwies:

„I expect that we may come to a new respect for deterrence. If Iran should, despite every diplomatic effort to prevent it, acquire a few nuclear weapons, we may discover again what it is like to be the deterred one, not the one doing the deterring. (I consider us - NATO - as having been deterred from intervening in Hungary in 1956 and Czechoslovakia in 1968.) I also consider it crucial that Iran learn to think, if it has not already, in terms of deterrence".

Zentral für die Absenz eines Nuklearkrieges in den Dekaden nach Hiroshima ist nach Schelling also, dass die Bedrohten wie die Drohenden die Logik dieser Waffen verstehen. Doch seit dem Ende der 1950er Jahre, als die moderne rationalistische Abschreckungstheorie entwickelt wurde, haben unterschiedlichste Kritiker auf die Grenzen solcher Empfehlungen verwiesen. Die ursprünglichen Anfechtungen waren durch die Furcht motiviert, dass der mit diesem Ansatz verknüpfte 
Anspruch, ,die Bombe zu denken', gerade das Konfliktrisiko schüre. Besonders drastisch meinte Green (1966, S. 225), „(...) deterrence theory justifies the indiscriminate killing of innocent persons under certain circumstances”. Moderater fiel die Kritik des Psychologen Anatol Rapoport (1964, S. 166, Kursivsetzung im Original) aus, der schon früh die Grundlagen der Abschreckungstheorie kritisierte und auf die Notwendigkeit einer psychologischen Fundierung dieses Ansatzes verwies:

„...any research which can seriously claim to be directed toward the prevention of war or especially toward the establishment of peace must be essentially concerned with psychological matters, with man, his motivations and social order".

Dieser Aufforderung kommen wir in diesem Aufsatz nach und überprüfen in einem spieltheoretisch fundierten Experiment, ob in Abschreckungssituationen bestimmte Handlungskontexte oder Individuen mit besonderen Dispositionen die Eskalation schüren. Dabei fokussieren wir besonders auf die Faktoren Zeitdruck und Überoptimismus, die verschiedenste Forscher in der jüngsten Zeit mit einer erhöhten Eskalationsbereitschaft in Verbindung gebracht haben. So zeigen Blendin und Schneider (2012) in einem Groupthink-Experiment, dass Zeitdruck als situativer Stressor die Entscheidungsqualität mindert, während Cortisol und damit ein biologischer Stressor keinen systematischen Effekt ausübt. Johnson et al. (2012) und andere haben ferner gezeigt, dass ein übersteigertes Selbstvertrauen, das wir auf die Formel des ,Überoptimismus' bringen, das Eskalationsrisiko erhöht; diese Tendenz begründen Johnson und Fowler (2011) evolutionstheoretisch. Selbstüberschätzung - der Glaube besser zu sein, als man tatsächlich ist - gilt als eine der häufigsten kognitiven Verzerrungen in der Wahrnehmung von Individuen (Johnson u. Fowler 2011, S. 317).

Als weitere psychologische Korrelate der Abschreckung kontrollieren wir die Risikoscheue und den Narzissmus der Probanden. Krämer und Schneider (2003, siehe auch Schneider und Krämer 2004) zeigen, dass das Sicherheitsbedürfnis sowie antisoziale Neigungen das Verhandlungsverhalten beeinflussen. In verschiedenen Studien (z. B. Resick et al. 2009 oder Chatterjee u. Hambrick 2011) wird Risikoverhalten mit narzisstischen Persönlichkeitsstörungen in Verbindung gebracht.

Das Experiment evaluiert diese Thesen zum Einfluss von zwei situativen (Stress sowie eines risikofördernden Handlungsrahmens) und von drei individuellen Faktoren (Selbstüberschätzung, Risikoaversion und Narzissmus) auf das Verhalten in Abschreckungssituationen. Konkret untersuchen wir auf der Grundlage eines Krisenverhandlungsspiels die Bereitschaft eines Kontrahenten, eine Forderung eines Gegners abzulehnen und damit eine Eskalation zu riskieren. Die Probanden sind dabei im Experiment unsicher darüber, ob ein Zurückweisen oder ein Eingehen auf das Verlangen der Gegenseite die adäquate Antwort ist. Um den möglichen Einfluss von Stress zu erfassen, vergleicht das Experiment zwei Experimentalgruppen miteinander: Im Stress-Treatment entscheiden die Spieler unter Zeitdruck, in der Kontrollgruppe ohne. Damit wir den Effekt eines eskalationsfördernden Handlungsrahmens erfassen können, konfrontieren wir die Probanden mit unterschiedlichen Aussichten auf Gewinn im Eskalationsprozess. In einem Szenario 
hängt die Gewinnwahrscheinlichkeit vom Abschneiden in einem Test bestehend aus zehn gängigen IQ-Testfragen ab, im alternativen Spielaufbau sind die Gewinnaussichten zufällig. Um schließlich den Einfluss von Persönlichkeitsmerkmalen auf das Abschreckungsverhalten zu evaluieren, erheben wir zum einen mithilfe von Einschätzungsfragen die Variable ,Überoptimismus‘. Dieses Konzept erfasst, ob sich die Person tendenziell selbst überschätzt. Die Variable „Risikoaversion“ operationalisieren wir über die Wahl zwischen einer sicheren oder unsicheren Lotterie mit identischem Erwartungswert, und für die Messung von ,Narzissmus greifen wir auf ein einschlägiges Persönlichkeitsinventar zurück, dem wir zwei unterschiedliche Dimensionen dieser individuellen Disposition entnehmen.

Die statistische Auswertung ergibt, dass das Eskalationsverhalten von situativen Faktoren wie auch der Persönlichkeit der Entscheider abhängt. Zum einen erhöht der Überoptimismus die Eskalationsbereitschaft eines Spielers, wenn die Gewinnwahrscheinlichkeit von der eigenen Fähigkeit abhängt. Bei zufälliger Gewinnwahrscheinlichkeit zeigt sich hingegen, dass Risikoaversion negativ mit der Eskalationsbereitschaft korreliert. Dies demonstriert nach unserem Dafürhalten, dass Persönlichkeitsmerkmale das Abschreckungsverhalten im Kontext von bestimmten Anreizen beeinflussen. Eine der Narzissmus-Skalen ist ebenfalls mit einer erhöhten Eskalationsbereitschaft verbunden. Zum anderen zeigen wir in Abgrenzung zur Groupthink-Literatur (Janis 1971; Blendin u. Schneider 2012), dass neben der individuellen Risikoaversion Zeitdruck und damit ein weiterer situativer Faktor die Eskalationsbereitschaft dämpft. Wir argumentieren abschließend, dass eine realistische Neuversion der Abschreckungstheorie das von uns beobachtete Zusammenwirken von Entscheidungssituation und Persönlichkeit berücksichtigen sollte.

\section{Determinanten des Abschreckungserfolgs und -misserfolgs}

Abschreckung ist nach den gängigen Definitionen der Versuch eines potentiell Angegriffenen, einem Aggressor durch die Androhung von Gewalt den Anreiz zum Angriff zu nehmen:

„A is expected to take some action $\mathrm{x}$ whose consequences $B$ does not like. $B$ therefore announces that if $A$ does $\mathrm{x}, B$ will take some action y whose consequences $A$ does not like (...). If so, $B$ can be said to have deterred $A$ from doing x" (Wagner 1992, S. 115).

Während erste Abschreckungsmodelle noch der Logik der kooperativen Spieltheorie folgten und dabei etwa das Feiglingsspiel (Chicken Game) zum Einsatz kam, hielt besonders über die Vorarbeiten von Schelling $(1958,1960)$ die nicht-kooperative Variante dieser formalen Theorie Einzug in die Literatur. Wesentlich für diese dynamischen Modelle ist die Einsicht, dass der Erfolg der Abschreckungsbemühungen des ,Verteidigers' von der Glaubwürdigkeit einer Drohung zu einem Gegenschlag abhängt.

In einer Welt mit Nuklearwaffen erwiesen sich militärische Doktrinen, die mit einem atomaren Gegenschlag drohen als Antwort auf einen aggressiven Schritt der Gegenseite, als Rohrkrepierer, da sie den eigenen Suizid im sprichwörtlichen 
Sinne von ,lieber tot als rot voraussetzen. Glaubwürdig ist für Schelling in einer solchen Situation eine Strategie des Brinkmankship, die nach der Formel „a threat that leaves something to chance" ein Restrisiko dem Zufall überlässt (Schelling 1960, S. 187ff.). Die Glaubwürdigkeit einer solchen Drohung hängt wesentlich vom Einsatz ab, mit dem sich ein Verteidiger für seine Position in solch impliziten Verhandlungen einsetzt: je größer seine Bereitschaft, die Kontrolle über die Eskalation abzugeben, desto stärker und glaubwürdiger ist seine Drohung. Um Erfolg zu haben, muss ein Verteidiger also darauf setzen, dass die andere Seite ihn als ,irrational' oder zumindest risikobereit wahrnimmt.

Der Abschreckungserfolg ist damit in der Brinkmanship-Perspektive nicht mehr abhängig vom Wettlauf um immer größere militärische Kapazitäten, sondern stellt einen Wettkampf um Risikobereitschaft dar (vgl. Jervis 1979; Powell 1987; Schelling 1960, 1966). Entscheidend für den Abschreckungserfolg ist also nur, ob die Signale des Verteidigers, auf der Eskalationsleiter eine Stufe weiter zu gehen, falls die Gegenseite den Konfrontationskurs fortsetzt, diese zum Einlenken bewegen. Schellings Ansatz setzt sich damit vom klassischen realistischen Gleichgewichtsdenken ab, wonach militärische Kapazitäten über den Abschreckungserfolg entscheiden (Zagare 1996). Vielmehr sind die Signale entscheidend, die Verteidiger und Angreifer über ihre Eskalationsbereitschaft austauschen. Damit hat Schelling das Werk von Jervis (1976) und anderen beeinflusst, die auf die kognitiven Probleme hinwiesen, die Botschaften der Gegenseite korrekt zu interpretieren.

Schellings Modelle wurden seit den 1980er Jahren weiter entwickelt und nahmen dabei die Form von ,Krisenverhandlungsspielen' an, in denen die Kontrahenten unsicher über die Entschlossenheit der Gegenseite sind, die Eskalation weiterzutreiben (Zagare u. Kilgour 2000). Der Ansatz hat dabei unter anderem auch der These des Demokratischen Friedens eine theoretische Fundierung verliehen, war es doch auf der Grundlage von Krisenverhandlungsspielen Fearon (1994) möglich zu zeigen, wie demokratische Regierungen ihre Unterstützer manipulieren, um ihre Gegenschlagsdrohung glaubhafter zu machen.

Gegner der modernen Theorie der rationalen Abschreckung haben seit der Genese dieses Ansatzes darauf hingewiesen, dass die Modellwelt sich nicht auf reale politische Abschreckungssituationen übertragen lasse und dass die handelnden Akteure die Rationalitätsannahmen nicht erfüllen könnten, die aus den spieltheoretischen Grundlagen folgen. Am leichtesten zu widerlegen ist die Fundamentalkritik, wie sie der Icherzähler in einem Roman des Literaturnobelpreisträgers Coetzee (2008, S. 20) anbringt, dass sich terroristische Organisationen im Gegensatz zu den Kontrahenten des Kalten Krieges nicht durch die prohibitiven Kosten von Attentaten abhalten ließen:

„The Islamist terrorists, on the other hand, are nothing about survival (...). Nor do such terrorists follow the rationalist calculus of costs and benefits: to deal a blow to God's enemies is enough, the cost of that blow, material or human, is unimportant."

Diese Interpretation übersieht, dass Selbstmordattentäter nur Instrumente der Planer der Anschläge sind und dass diese Organisatoren ihre Terrorkampagnen zum Erreichen von politischen Zielen und nicht als Selbstzweck durchführen. 
Ähnliches gilt für die bekannte metaphorische Kritik Halperins (1987, S. 85, 113), wonach Brinkmanship das Monster erst erschaffe, das es zu bekämpfen vorgebe. Demnach sei die Abschreckungstheorie und die auf ihr aufbauende Sicherheitspolitik eine "doomsday machine linked to a roulette wheel". Doch Unsicherheit ist für Abschreckungssituationen konstitutiv, und der Verzicht, ,die Bombe zu denken' und sich entsprechend mit ihr intellektuell auseinander zu setzen, erhöht wohl eher das Konfliktrisiko, als es zu mindern.

In einem Sonderheft der Zeitschrift World Politics argumentierten Lebow und Stein (1989, S. 224) ebenso fundamental, dass rationale Abschreckungstheorien „(...), theories' about nonexistent decision makers operating in nonexistent environments" seien. Grundlage für diese Zurückweisung waren Fallstudien, welche wie bei Janis' (1972, 1971) Fallstudien zum Gruppendenken-Phänomen fast ausschließlich gescheiterte Abschreckungsbemühungen untersuchten und damit einem Induktionsschluss unterlagen (Achen und Snidal 1989).

Eine weitere, wenn auch stärker fokussierte Grundsatzdebatte entspannte sich jüngst zum Konzept der „Publikumskosten“ („audience costs“), das Fearon (1994) in die Debatte einführte. Publikumskosten stellen nach Tomz (2007, S. 871) den innenpolitischen Preis dar, den politische Führer in einer Krise für das Entwickeln von Drohungen und das Zurücknehmen von ihnen zu zahlen bereit sind. Snyder und Borghard (2011) verweisen darauf, dass Publikumskosten unter anderem in Krisen deshalb eine geringe Bedeutung hätten, weil demokratische Regierungen sich nicht eindeutig auf eine Abschreckungsdrohung verpflichten ließen, um bei einem Scheitern des Konfrontationskurses innenpolitisch noch ihre Haut retten zu können. In einem Symposium zu Trachtenbergs (2012) historischer Analyse, in welcher der amerikanische Historiker wenige bis keine Belege für Fearons Kausalmechanismus zu glauben fand, nahm Mercer (2012, S. 402) noch einmal die Standardkritik an der Abschreckungstheorie auf:

„Audience cost arguments seem not to exist in practice because rational people do not think the way rational choice theorists think they should think."

So fruchtbar solche Zuspitzungen manchmal sein mögen, so wenig konstruktiv sind sie in anderen Fällen, da die Kritiker bis jetzt keine Alternative vorgelegt haben, sieht man von den Versuchen ab, die „Prospect Theory“ von Kahneman und Tversky (1979) für die Analyse von Abschreckungssituationen fruchtbar zu machen (z. B. Levy 1992a, b; Farnham 1994). Dazu kommt, dass die Hypothesen, die sich aus Krisenverhandlungsspielen ableiten lassen, durchaus einige empirische Unterstützung erhalten (z. B. Tomz 2007).

Ernster zu nehmen sind die Hinweise in der Nachfolge zu Rapoport (1964), dass die Abschreckungstheorie einer psychologischen Fundierung bedürfe. In seinem Anschluss forderten etwa Jervis (1988) oder Stein (1988), dass bei der Analyse des politischen Entscheidungsverhaltens verstärkt die individuelle Bedrohungswahrnehmung eine Rolle spielen soll. Spätestens seit Janis (1971, 1972) wissen wir überdies um die Bedeutung von krisenimmanenten Faktoren wie Zeitund Gruppendruck. Im Folgenden diskutieren wir, welche psychologischen Korrelate die Eskalationsbereitschaft in Abschreckungssituationen erhöhen bzw. dämp- 
fen könnten. Grundsätzlich sind wir dabei der Überzeugung, dass der mögliche Nachweis von nicht-rationalen Determinanten des Abschreckungsverhaltens eher zu einer Korrektur der Theorie und nicht zu einer Zurückweisung der rationalistischen Postulate führen sollte. Unser Streben richtet sich also nach einer realistischen Theorie, die die Entscheider und die besondere Situation berücksichtigt, in der diese stehen. Die Theorie der rationalen Abschreckung und die Psychologie müssen sich also in unserer Perspektive nicht widersprechen, sondern eher ergänzen. In diesem Sinne verstehen wir auch Mercer (2010, S. 13), wonach Emotionen und damit nicht-rationale Faktoren die Glaubwürdigkeit einer Gegenschlagsdrohung erst ermöglichten: „Imagining that emotion only interferes with analysis is wrong: someone deprived of all emotion becomes vacuous, not neutral". Andere Skeptiker der rationalistischen Abschreckungspostulate wie Jervis $(1976,1988)$ haben deshalb ähnlich wie Mercer das Grundgerüst der dynamischen Abschreckungsanalyse übernommen, verweisen aber zum Beispiel auf die Probleme, die Signale der Gegenseite in einer konkreten Eskalation zu entschlüsseln.

Unsere theoretische Erweiterung der rationalen Abschreckungstheorie unterscheidet zwischen dem Kontext, in dem Abschreckung stattfindet, und den individuellen Dispositionen der Entscheidungsträger. Um den Vorwurf des theoretischen Eklektizismus zu vermeiden, beschränken wir uns dabei in der Diskussion vorwiegend auf Faktoren, die in der jüngeren Abschreckungsdiskussion und der experimentellen Verhandlungsliteratur eine zentrale Rolle einnehmen: Stress, Framing und Selbstüberschätzung. Wir kontrollieren zusätzlich, ob Persönlichkeitsmerkmale wie Narzissmus und Risikoscheue den Zusammenhang beeinflussen. Während der Stress und das Framing zu den situativen Faktoren gehören, ordnen wir die anderen Korrelate den individuellen Dispositionen zu.

Situative Faktoren: In der Regel werden Entscheidungen über Kooperation oder Eskalation in einer Abschreckungssituation unter Stress getroffen. Die sozialpsychologische Forschung hat mehrfach festgestellt, dass Stress in Form von Zeitdruck die Entscheidungsfindung beeinflusst (vgl. Ben Zur u. Breznitz 1981; De Dreu 2003; Svenson 1981; Svenson u. Maule 1993). Auch in der Politik ist Zeitdruck ein Faktor, der sowohl als konfliktschürender Stressor wie auch als kooperationsfördernde Verhandlungstaktik interpretiert wird. Verhandlungsführer erzeugen oft Zeitdruck, um Kompromisse zu erreichen bzw. zu erleichtern. Der amerikanische Präsident Jimmy Carter soll während der Verhandlungen zwischen Ägypten und Israel in Camp David 1978 auf diese Strategie gesetzt und dadurch eine Einigung zwischen den Kontrahenten bewirkt haben (Carnevale u. Lawler 1986, S. 637). Dennoch verringert Zeitdruck die Chance, dass sich Streithähne in bilateralen Verhandlungen gütlich einigen (Pinfari 2011; Carnevale u. Lawler 1986; Carnevale et al. 1993).

Eine zentrale Rolle spielt der Stress in der Konfliktforschung spätestens seit Janis' (1971) grundlegendem Beitrag zum Phänomen des Gruppendenkens. Zu berücksichtigen ist dabei allerdings nach Janis und Mann (1977), dass der $\mathrm{Zu}$ sammenhang zwischen Stress und Entscheidungsqualität kurvilinear ist und dass sich bei einem mittleren Stressniveau die Informationsverarbeitung verbessert, ein Zusammenhang, den Brecher (1980) anhand zweier Fallstudien (Israels Entscheidungskrisen 1967 und 1973) bestätigt. Hermann (1979, S. 29) ist skeptischer und 
weist auf die Schwierigkeiten hin, den Umschlagepunkt zu bestimmen, ab dem Stress das Verhalten beeinträchtigt.

Die jüngere experimentelle Forschung liefert aber genügend Belege für Janis' (1971) ursprüngliche Skepsis. So konnten die Autoren in einem Groupthink-Experiment zeigen, dass „Stress in Form von Entscheidungsdruck durch Zeitdruck und Tonsignal im Schnitt die Entscheidungsqualität mindert" (Blendin u. Schneider 2012, S. 76). Andere Studien bestätigen diesen Zusammenhang. Das Computerexperiment von Ibañez, Czermak und Sutter (2009) demonstriert ferner, dass gerade bei unerfahrenen Spielern das Suchverhalten suboptimal ist. Wir vermuten aufgrund dieser verschiedenen Befunde, dass Zeitdruck die Gefahr einer Eskalation steigert.

Die Chance, in einer Krise bestehen zu können, ist indes durch die Gewinnaussichten vermittelt. Im konkreten Experiment unterscheiden wir zwei Entscheidungssituationen, um Framingeffekten auf die Spur zu kommen. In einem ersten Experiment teilen wir den Probanden mit, dass ihr Entscheidungsverhalten von ihrem Erfolg bzw. erzielten Ranglistenplatz in einem Wissenstest abhängt. Die zweite Möglichkeit ist dabei, dass ihre Erfolgschance zufälliger Natur ist. Wir erwarten, dass sich der Einfluss der Erklärungsfaktoren unterscheidet, je nachdem, ob sich ein Akteur in den entsprechenden ,Rang'- oder ,Zufallsspielen“ befindet.

Framing-Effekte, wie wir sie hier postulieren, spielen in der empirischen Abschreckungsforschung eine große Rolle. So haben verschiedenste Studien auf der Grundlage der Prospect-Theorie (Kahneman u. Tversky 1979) nachzuweisen versucht, dass das Risikoverhalten in einer Verlustsituation ausgeprägter sein sollte als in einem positiven Entscheidungsrahmen. So sollte nach Levy (1992b, S. 289) ein Staat bei drohenden Verlusten eher zu riskanten Entscheidungen neigen, was sich negativ auf den Abschreckungserfolg auswirken sollte. Umgekehrt sollte der Abschreckungserfolg wahrscheinlicher sein, wenn der Angreifer risikoavers ist und den Status quo hoch bewertet. Ähnlich erwarten wir, dass der Entscheidungsrahmen den Einfluss von Stress auf die Eskalationsbereitschaft moderiert. Im Rangspiel sind sich die Spieler sicher, dass sie die Situation erfolgreich bestehen können.

Wir erwarten deshalb unter Stress einen größeren Effekt im Rang- im Vergleich zum Zufallsspiel.

H1: Zeitdruck erhöht die Eskalationsbereitschaft eines Verteidigers in einer Situation akuter Abschreckung. Dieser Effekt ist größer im Rang-im Vergleich zum Zufallsspiel.

Selbstüberschätzung und weitere psychologische Erklärungsfaktoren: Zu Situationen, die suboptimales Verhalten fördern, kommen individuelle Dispositionen als mögliche Konfliktverstärker. Zentral ist dabei das Konzept der Selbstüberschätzung. Darunter ist eine kognitive Verzerrung zu verstehen, die verschiedene Ursachen und Ausprägungen annehmen kann (Pallier et. al 2002). Wir konzentrieren uns hierbei auf das Phänomen des Überoptimismus, das in der letzten Dekade vermehrt für Eskalationstendenzen verantwortlich gemacht wurde (Johnson et al. 2012; Johnson u. Fowler 2011; Johnson 2004), als einer zentralen Komponente der Selbstüberschätzung. Unter „Overconfidence“ wird demnach eine positive Il- 
lusion in die eigenen Fähigkeiten, eine zu optimistische Einschätzung von Gefahren oder die Unterschätzung von Risiken verstanden (Johnson 2004).

In der experimentellen Forschung zeigt sich, dass Akteure in Verhandlungsspielen eher mit Zugeständnissen durch den Gegner rechnen, wenn die eigenen Erfolgswahrscheinlichkeiten höher eingeschätzt werden (Kochan u. Jick 1978). Übertriebener Optimismus in die eigenen Fähigkeiten korreliert nicht nur mit der Wahrscheinlichkeit einer Einigung, sondern auch mit der Einigungsanstrengung der Parteien (vgl. Carnevale u. Pruitt 1992, S. 557). Johnson et al. (2006) simulieren ein Krisenverhandlungsspiel, in dem die Teilnehmer die Rolle eines staatlichen Führers einnehmen. Sie finden einen Zusammenhang zwischen Überoptimismus und aggressiven Strategien, jedoch ist der kausale Zusammenhang aufgrund der Komplexität des Entscheidungsspiels nicht eindeutig. Johnson und Fowler (2011) begründen die Persistenz dieser Ergebnisse durch den Vorteil, den diese spezielle Erscheinungsform der Selbstüberschätzung in einer evolutionären Perspektive aufweist (Johnson u. Fowler 2011). In internationalen Krisen begünstigen Überoptimismus und fehlerhafte Einschätzungen der Akteure eine Bereitschaft zum Einsatz militärischer Mittel und verringern die Chancen auf friedliche Einigungen und kooperatives Verhalten (Kahneman u. Renshon 2007, S. 34). Johnson et al. (2012) bestätigen diese Tendenzen.

Johnson (2004, S. 5) bringt den Zusammenhang zwischen der überoptimistischen Einschätzungsverzerrung und der Wahrscheinlichkeit der Kriseneskalation auf die Formel: „(...) overconfidence contibutes to causing war“. Wir übernehmen diese Hypothese für unsere Experimentalstudie und postulieren gleichzeitig einen weiteren Framingeffekt. So gehen wir davon aus, dass diese Tendenz größer ist in Situationen, in denen der Erfolg einer Konflikteskalation von den eigenen Fähigkeiten abhängt, wofür in unserem Experiment das Szenario des Rangspiels steht.

H2: Überoptimistische Persönlichkeiten neigen in einer Situation akuter Abschreckung eher zu Konflikteskalation. Dieser Effekt sollte besonders im Rangspiel auftreten.

Im Zusammenhang mit Selbstüberschätzung in Form von überoptimistischen Selbsteinschätzungen kontrollieren wir das Persönlichkeitsmerkmal Narzissmus. Es reicht von einer Tendenz, sich selbst übertrieben positiv wahrzunehmen (,positive illusion“), bis zu einer Wahrnehmung des eigenen Selbsts als großartig und einzigartig (Gabriel et al. 1994; Raskin u. Hall 1981). Ein narzisstisches Persönlichkeitskonstrukt, welches sich durch Selbstüberschätzung und ein dominant-aggressives Interaktionsverhalten auszeichnet, wird zwar nicht als klinisch, aber dennoch als problematisch eingeschätzt (Schütz et al. 2004, S. 203).

Zook (2000) beschwört die Gefahr eines Nuklearkrieges zwischen Indien und Pakistan mit einem kulturellen Narzissmus als Erklärungskonzept. Sozialpsychologische Studien haben fernab von solchen Spekulationen gezeigt, dass hoch-narzisstische Top-Manager besonders dann risikobereit sind, wenn sie sich auf positive Medienreaktionen stützen können (Chatterjee u. Hambrick 2011). Zeigler-Hill, Enjaian und Essa (2013) zeigen überdies, dass unter Männern Narzissmus mit sexueller Aggression einhergeht, während Jones und Paulhus (2010) demonstrieren, dass gerade Narzissten aufgrund einer Provokation eher mit Aggression re- 
agieren als Individuen, bei denen diese Persönlichkeitsstörung nicht vorhanden ist. Aufgrund dieser Regelmäßigkeiten können wir davon ausgehen, dass narzisstische Persönlichkeiten in einer Konfliktsituation unabhängig von der spezifischen Abschreckungssituation den Konflikt tendenziell weiter treiben.

$\mathrm{Zu}$ beachten ist dabei allerdings, dass nur bestimmte Formen des Narzissmus die Neigung zu aggressivem Verhalten beeinflussen. So weisen Pincus und Lukowitsky (2010) nach, dass scheue Narzissten aufgrund ihrer Hypersensitivität gegenüber Kritik eher Auseinandersetzungen meiden, während psychopatische Narzissten eher mit Neid und Aggression reagieren. Auch Evaluationen zentraler Messinstrumente wie etwa des Narcissistic Personality Inventory (NPI) legen nahe, dass Narzissmus ein mehrdimensionales Konzept ist (Ackerman et al. 2011).

H3: Narzisstische Persönlichkeiten neigen in einer Situation akuter Abschreckung eher zu Konflikteskalation.

Die Erwartungsnutzentheorie wie die Prospect-Theorie stimmen darin überein, dass Entscheidungen unter Unsicherheit durch die Einstellung der Akteure zu Risiken beeinflusst werden. Vor diesem Hintergrund überrascht es wenig, dass Risikoaversion bzw. - freudigkeit einen großen Stellenwert haben in der Diskussion zur Effektivität der Abschreckung. So erklärt etwa Farnham (1992, siehe auch Farnham 1994) in einer Fallstudie zur Sudetenkrise, dass Präsident Roosevelt im Laufe der Verhandlungen zum Münchner Abkommen die Entscheidung über einen möglichen Krieg in einen möglichen Verlust umwandelte, den er fortan vermeiden wollte, auch wenn dies mit neuen Risiken einherging. Mercer (2010) führt ferner unter anderem Saddam Husseins Kurs vor dem 2. Golfkrieg auf die Fehlwahrnehmung zurück, wonach die Vereinigten Staaten durch eine übertriebene Risikoaversion geleitet seien. Dazu kommt experimentelle Evidenz, welche die Einstellung zur Entscheidungsfindung unter Unsicherheit als Mitursache für sichere oder risikoreichere Entscheidungen sieht. So zeigen etwa Thaler et al. (1997), dass die Tendenz zu kurzfristiger Verlustaversion und damit zu einem Konzept der Prospect-Theorie mit konservativen Anlageentscheidungen einhergeht. In einem Experiment zu Mechanismen der fairen Teilung stellen Krämer und Schneider (2003) für risikoscheue Probanden fest, dass sie eher Verfahren wählen, die sie aus dem Alltag kennen. Die Prospect-Theorie lässt darüber hinaus erwarten, dass der Handlungsrahmen eine Rolle spielt. So sollte im Rangspiel die Tendenz zur Deeskalation, die mit Risikoaversion einhergeht, ausgeprägter sein als im Zufallsspiel.

H4: Risikoaverse Persönlichkeiten vermeiden in einer Situation akuter Abschreckung den Konflikt weiter eskalieren zu lassen. Diese Tendenz sollte im Zufallsspiel ausgeprägter sein.

\section{Forschungsdesign und Methode}

In unserem Experiment gehen wir von einem Szenario aus, in dem die sog. generelle Abschreckung bereits gescheitert ist und in dem der erste Schritt im zugrundeliegenden Krisenverhandlungsspiel - das Formulieren einer monetären Forde- 
rung durch den Angreifer - bereits erfolgt ist. Der Gegenpart zu diesem Aggressor, der Verteidiger, muss daher entscheiden, ob er diese Forderung annimmt oder ob er eine Gegenforderung stellt.

Formaltheoretisch kann die rationale Entscheidung aus den Wahrscheinlichkeitsparametern des Modells hergeleitet werden, das wir in einem Online-Anhang näher skizzieren. Dem nicht-kooperativen Spiel liegt die Annahme zugrunde, dass der Verteidiger unsicher über die Eskalationsbereitschaft der Gegenseite ist. In anderen Worten weiß dieser Spieler nicht, wie glaubwürdig die Drohung zu einer weiteren Eskalation ist.

Handlungsoptionen und Auszahlungen: Im Experiment wurden je zwei Versuchspersonen einander zugeteilt und anonym über zwei Rechner miteinander verbunden. Die Situation setzt mit einer Forderung von Spieler 1 - dem Angreifer - ein. Spieler 2 - der Verteidiger - kann auf die Forderung eingehen und eine kooperative Strategie wählen oder die Forderung ablehnen und damit eskalieren. Untersucht wird konkret, welche Faktoren die Eskalationsbereitschaft des Verteidigers beeinflussen.

\section{Abbildung 1: Grundstruktur Abschreckungsexperiment}

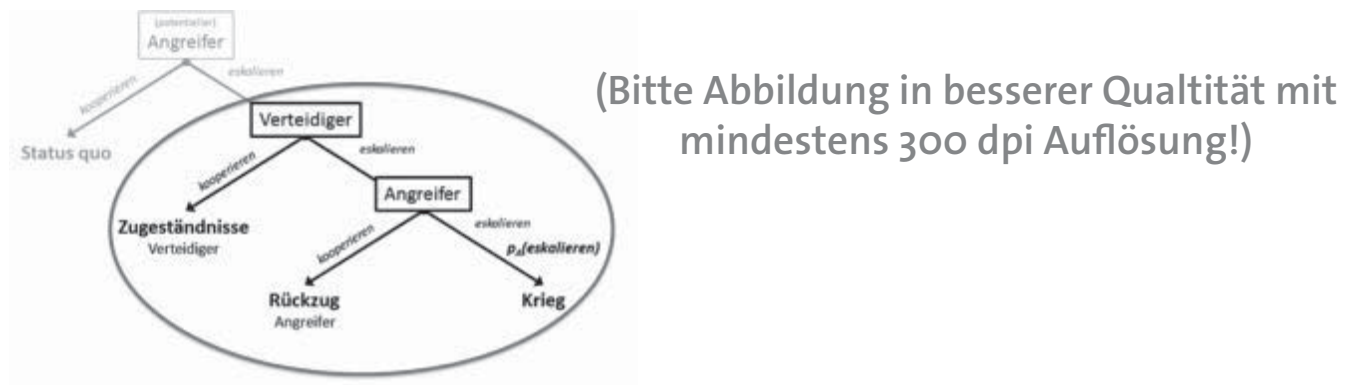

Abbildung 1 stellt die Grundstruktur des Experiments dar, das auf einem Modell von Zagare und Kilgour (2000, Kap. 5) beruht. Der Verteidiger entscheidet unter Unsicherheit, wobei die kritische Größe die Wahrscheinlichkeit $p_{A}$ (eskalieren) ist: Die optimale Entscheidung des Verteidigers hängt davon ab, wie wahrscheinlich der Angreifer eskalieren wird. Die Auszahlungen im Experiment wurden so gestaltet, dass der Verteidiger - im Folgenden Spieler $2-$ bei $p_{A}=0,5$ zwischen den beiden Optionen kooperieren und eskalieren indifferent ist. Damit ergibt sich als Nullhypothese des Verteidigerverhaltens: Ohne externe Einflussfaktoren ist zu erwarten, dass beide Entscheidungen gleich häufig auftreten müssten. Die Entscheidung von Spieler 2 wird über die Variable Eskalationsbereitschaft erhoben, die den Wert 1 annimmt, wenn Spieler 2 die Forderung ablehnt, und 0, wenn er sie annimmt.

Framingeffekte: Abweichungen vom oben hergeleiteten Gleichgewicht erwarten wir teilweise in Abhängigkeit zum Handlungsrahmen. Es werden zwei Entscheidungssituationen unterschieden: In einem ersten Experiment teilen wir den Probanden mit, dass ihre Gewinnwahrscheinlichkeit von ihrem Abschneiden in einem Wissenstest - ihrem erzielten Ranglistenplatz - abhängt. Im zweiten Setting ist die Erfolgschance zufälliger Natur. Wir erwarten, dass sich der Einfluss der Erklärungsfaktoren unterscheidet, je nachdem, ob sich ein Akteur in den entspre- 
chenden ,Rang'- oder ,Zufallsspielen` befindet. Im Zufallsspiel wird der im Konflikt siegende Spieler zufällig ermittelt. Die Wahrscheinlichkeiten $p A$ und $p V$ sind demnach beiden Spielern bekannt und die Entscheidung wird unter Unsicherheit getroffen. Ohne Verzerrungen sollte davon ausgegangen werden, dass hier gemäß der Erwartungsnutzentheorie entschieden wird. Im Rangspiel hingegen hängen sie von im Vorfeld ermittelten Ranglistenplätzen der Spieler ab, welche unbekannt sind. Die Entscheidungssituation hat also eine zusätzliche Quelle der Unsicherheit, da die Wahrscheinlichkeiten $p A$ und $p V$ von den Spielern eingeschätzt werden müssen. Entsprechend ihres Abschneidens in einem Fragentest zu Beginn des Experiments, bestehend aus 10 IQ-Testfragen, wurden die Probanden in eine eindeutige Reihenfolge gebracht. Je mehr Wissensfragen richtig beantwortet wurden und je schneller diese beantwortet wurden, desto höher rangierte der/die einzelne Proband/in. Wir gehen davon aus, dass sich die Experimentalteilnehmer hier selbst einschätzen und eine Vorstellung davon bilden, wie hoch ihre individuelle Gewinnwahrscheinlichkeit ist.

Spieler 2 wird zu Beginn einer Runde darüber informiert, welche Variante gespielt wird, nicht aber, welche konkrete Auszahlung ihn im Konflikt erwarten würde. Es wird also angenommen, dass er eine Einschätzung über die Gewinnwahrscheinlichkeit bildet. In der Variante Zufallsspiel ist die Gewinnwahrscheinlichkeit exogen $(p A=0,5)$. Da dieser a priori-Wert dem Indifferenzschwellenwert des Verteidigers entspricht, kann gemäß der Erwartungsnutzentheorie in dieser Variante angenommen werden, dass Spieler 2 eine gemischte Strategie spielt und beide Aktionen mit gleicher Wahrscheinlichkeit gewählt werden. Anders ist hingegen die Logik beim Rangspiel. Wie im Online-Anhang gezeigt, gibt es formal und unter vollkommener Information nur eindeutige Strategien: Die Spieler der oberen Rangfolgenhälfte sollten eskalieren, die Spieler der unteren kooperieren. Indifferent ist lediglich der Medianspieler.

Zeitdruck: Während einer vorbereitenden Aufgabe, die aus der Beantwortung von Wissensfragen bestand, wurde den Teilnehmern im Zeitdruck-Treatment ein Countdown von 15 Sekunden angezeigt, der mit dem Geräusch einer tickenden Uhr verbunden war. Nach Ablauf der Zeit erschien ein blinkendes Textfeld: „Bitte entscheiden Sie jetzt!!!“, und das Geräusch einer Sirene ertönte. Wenn eine Probandin diesem Treatment unterworfen war, hat die Variable den Wert 1, 0 für den anderen Fall.

In Experimenten, die auf einer ähnlichen Entscheidungssituation beruhen, zeigten sich unterschiedliche Effekte. Sutter, Kocher und Strauß (2003) stellen beispielsweise in einem Experiment zum Ultimatumspiel fest, dass enge zeitliche Beschränkungen die Ablehnungsrate von Angeboten erhöhen (Sutter et al. 2003). Mosterd und Rutte (2000) untersuchen die Rolle von Zeitdruck in einer reduzierten Verhandlungssituation, die strukturell einem Feiglingsspiel ähnelt. Offenbar haben Spieler, die auf sich selbst gestellt sind, unter Zeitdruck eine höhere Kooperationsbereitschaft, während Spieler, die eine Gruppe repräsentieren, eher zu aggressiven Entscheidungen neigen. Darüber hinaus muss davon ausgegangen werden, dass die Stress-Leistungs-Kurve konkav verläuft, also die Leistungsqualität im niedrigeren Stress-Niveau zunächst ansteigt und erst ab einem bestimmten Stressniveau beeinträchtigt wird (Hermann 1979). Es ist also nicht eindeutig, wel- 
cher Effekt von diesem Stresstreatment auf die Entscheidungen der Spieler erwartet werden kann. Mit Janis' (1971) Konzept des Groupthink, welches situativen Stress in der Form von Zeitdruck als wesentliche Ursachen für defizitäre Entscheidungen in der internationalen Politik annimmt, gehen wir hier davon aus, dass extreme verkürzte Entscheidungshorizonte die Einschätzung der Situation bei den Experimentalteilnehmern beeinflussen und die Wahrscheinlichkeit einer Konflikteskalation erhöhen.

Selbstüberschätzung: Selbstüberschätzung wird zu Beginn des Experiments über Einschätzungsfragen zum Wissenstest erhoben. Anhand von drei Einschätzungsfragen wird erhoben, ob der Spieler sich tendenziell besser einschätzt, als er tatsächlich war. Aus diesem Grund erfasst unser Indikator der Selbstüberschätzung den möglichen Überoptimismus der Probanden. Die drei Items sind an die in der Literatur diskutierten Überschätzungsarten angelehnt: (1) die Unfähigkeit, sich selbst adäquat einzuschätzen mit der Tendenz, sich besser einzuschätzen als man tatsächlich ist („calibration based overconfidence“: Gigerenzer et al. 1991, Keren 1991, Russo u. Schoemaker 1992), (2) die Positiv-Illusion, die sich darin zeigt, dass sich Menschen hinsichtlich der eigenen Fähigkeiten oder Leistungen sicherer sind, als es der Realität entspricht (Langer 1975, McKenna 1993, Weinstein 1980, Zakay u. Glicksohn 1992) und (3) der besser-als-der-DurchschnittEffekt („,better-than-average-effect“), der beschreibt, dass sich Menschen im Vergleich zu anderen tendenziell zu gut einschätzen (Alicke et al. 1995, Eiser et al. 2001, Svenson 1981). Da die drei Aspekte zugunsten der Eindeutigkeit (ja, nein) in der Messung stark reduziert wurden, haben wir eine Variable kodiert, die für Selbstüberschätzung den Wert 1 nur dann annimmt, wenn alle drei Aspekte auf Selbstüberschätzung schließen lassen.

Risikoaversion: In einem Beitrag von Holt und Laury (2002) wird Risikoaversion in Experimenten über die Wahl von mehr oder weniger sicheren Lotterie-Alternativen modelliert (Holt u. Laury 2002). Daran angelehnt wurde die Risikoneigung hier mit einer einfachen Auswahlfrage erhoben: „Wählen Sie eine Alternative: (1) 5,00 ECU sicher, oder (2) 10,00 ECU mit einer 50:50 Chance“. Alternative 1 steht für Risikoaversion, Alternative 2 für Risikoaffinität.

Narzissmus: In diesem Zusammenhang wurde für die Kontrolle von Narzissmus als Persönlichkeitsmerkmal hier das Narzissmus Inventar (NI) von Denecke und Hilgenstock (1989) verwendet, das für die Feststellung von unterschwelliger, leichter bis hin zu krankhafter Selbstüberschätzung ein valides Messinstrument darstellt (Denecke u. Hilgenstock 1989). Um der Mehrdimensionalität des Konstrukts Genüge zu tun, haben wir zwei Subskalen herausgegriffen: aus der Skala „klassisch narzisstische Persönlichkeit“ das „Größenselbst“, und aus der Skala „idealistisches Selbst“ die Subskala „Autarkie-Ideal“. Die Skala Größenselbst „entspricht dem nicht-klinischen Konzept von Narzissmus als Persönlichkeitskonstrukt am meisten“ (Schütz et al. 2004, S. 203), weswegen sie hier Verwendung findet. Im Gegensatz hierzu geht die Skala Autarkie-Ideal umgekehrt von der Auffassung eines bedrohten Selbsts aus, welches sich über narzisstische Einstellungen $\mathrm{zu}$ schützen versucht. Die insgesamt 20 Items wurden über Zustimmung oder Ablehnung bestimmter Aussagen mit einer Likert-Skala von 1 
(nicht zutreffend) bis 5 (völlig zutreffend) erhoben. Die Items werden für beide Skalen separat aufsummiert und der Durchschnittswert gebildet.

Ablauf des Experiments: Die Experimente fanden an sieben Sitzungen im Wintersemester 2011/2012 im Computerexperimentallabor LakeLab der Universität Konstanz statt. Das Experiment wurde mit z-Tree programmiert (Fischbacher 2007). Die Versuchspersonen wurden mit ORSEE, dem Online-Rekrutierungssystem für ökonomische Experimente, rekrutiert (Greiner 2004). Nach einer Teilnahme wurde die Person für weitere Sitzungen ausgeschlossen, um Sitzungseffekte zu vermeiden. Von den insgesamt 168 Teilnehmern waren 87 Männer und 81 Frauen, was einem Frauenanteil von 48\% entspricht. In drei zufällig ausgewählten Sitzungen wurde das Zeitdruck-Treatment (ZTT) angewandt, sodass 60 Personen im Zeitdruck-Treatment waren, 30 davon in der Rolle des Verteidigers (15 Frauen, 15 Männer). Das Durchschnittsalter betrug 22.27 Jahre, der jüngste Teilnehmer war 18, der älteste 30 Jahre alt. Es nahmen Studierende aller Fakultäten teil. Zunächst waren zehn Spielrunden geplant. Allerdings zeigte sich in der Feldphase, dass die komplexe Spielstruktur längere Wartezeiten für die Teilnehmer bedeutete, welche die ersten Teilnehmer in ihren Rückmeldungen als störend bezeichneten. Um die Ergebnisse hierdurch nicht zu beeinträchtigen, wurden schließlich nur noch fünf Runden gespielt. Die Daten der Runden 6-10 wurden in der Auswertung nicht berücksichtigt. Die Datenanalyse lässt darauf schließen, dass Ermüdungstendenzen die Entscheidungen schon nach drei Runden beeinflussen. Die Ergebnisse, die wir im nächsten Abschnitt berichten, beziehen sich daher auf jeweils drei und fünf Spielrunden.

Jeder Teilnehmer erhielt 5,00 Euro Aufwandsentschädigung plus eine Auszahlung in Abhängigkeit von den getroffenen Entscheidungen während des Experiments und in Abhängigkeit davon, welche Runde zur Auszahlung ausgewählt wurde. Es konnten bis zu 20,00 Euro zusätzlich erspielt werden. Pro Sitzung wurden jeweils die Hälfte der Teilnehmer zufällig zu Spieler 1 (Angreifer) und die andere Hälfte zu Spieler 2 (Verteidiger) gekürt. Diese Rolle blieb während des gesamten Experiments erhalten. Zu Beginn des Experiments wurden die Instruktionen laut vorgelesen. Die Informationen, die allen Teilnehmern zu Beginn des Experiments vorgelesen wurden, sind im Online-Anhang zu finden.

\section{Ergebnisse}

Dieser Artikel prüft, welche psychologischen Faktoren das Konfliktverhalten in einem akuten Abschreckungsfall beeinflussen. Dabei unterscheiden wir zwischen situativen Faktoren (Stress in Form von Zeitdruck, Framing in Form von unterschiedlichen Gewinnerwartungen) und Persönlichkeitsmerkmalen der Probanden (Überoptimismus, Risikoaversion, Narzissmus).

Wir erfassen dabei die Neigung, den Konflikt eskalieren zu lassen, über die Variable Eskalationsbereitschaft. Wie Abbildung 2 zeigt, übersteigt diese den erwarteten Wert von 0,5. Allerdings fällt sie von Runde 1 bis $4 \mathrm{ab}$, um in der Schlussperiode noch einmal nach oben zu schnellen. Es überlagern sich hier möglicherweise Lern- und Schlussrundeneffekte. 
Abbildung 2: Entwicklung der Eskalationsneigung über die fünf ersten Verhandlungsperioden

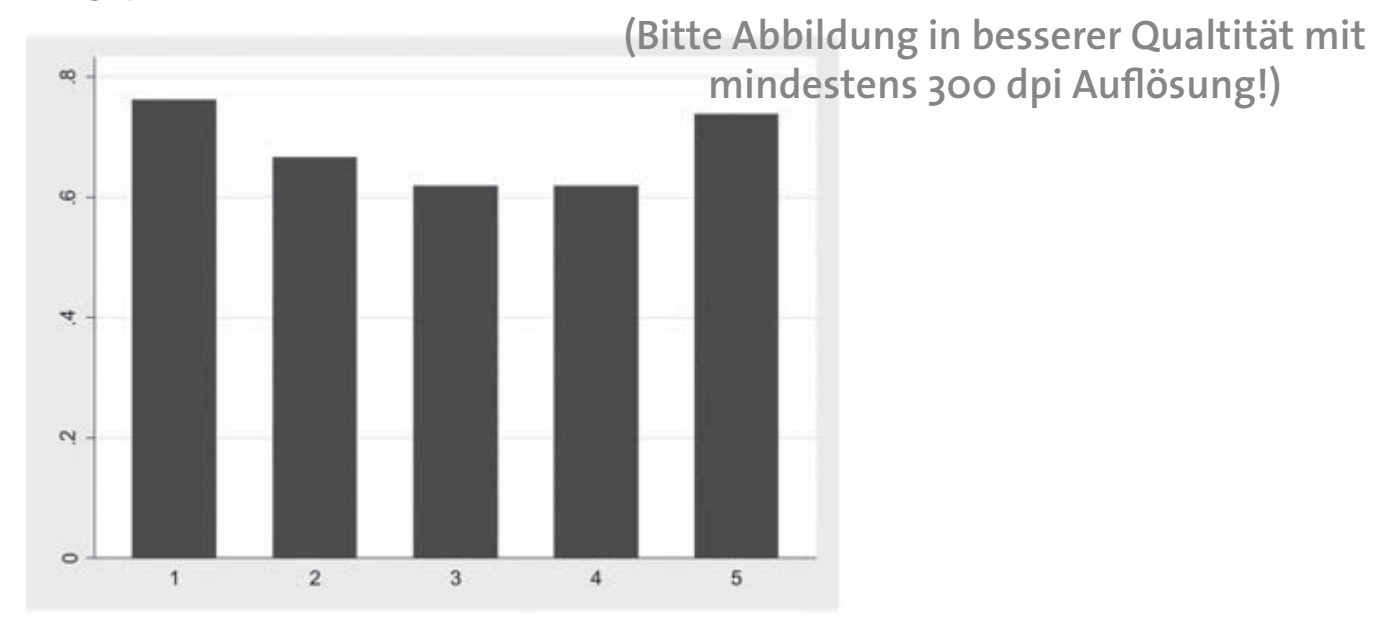

Das Abweichen vom erwarteten Durchschnittswert widerlegt die spieltheoretisch abgeleitete Nullhypothese, wonach die Probanden indifferent sein müssten zwischen den beiden Optionen, die Forderung des Angreifers zu akzeptieren oder sie abzulehnen und so den Konflikt eskalieren zu lassen. Dies wirft die Frage auf, ob sich das unterschiedliche Verhalten durch den Entscheidungskontext bzw. die individuellen Dispositionen erklären lässt. Dazu haben wir unterschiedliche logistische Modelle errechnet, die wir in Tabelle 1 ausweisen. Die Tests beziehen sich auf drei bzw. fünf Perioden. Wir unterscheiden drei Modelltypen. Zunächst präsentiert die Tabelle die Resultate für das Gesamtmodell, anschließend für das Zufallsspiel und am Schluss für das Rangspiel. Den Einfluss der unterschiedlichen Frames versuchen wir also über eine Teilung des Samples zu demonstrieren. Modelle 1, 5 und 9 erfassen den Einfluss des Zeitdruck-Treatments, Modelle 2, 6 und 10 eruieren den Effekt des Überoptimismus, Modelle 3, 7 und 11 berücksichtigen beide Variablen gleichzeitig, und die Modelle 4, 8 und 12 beinhalten die Kontrollvariablen (beide Aspekte des Narzissmus und Risikoaversion). 


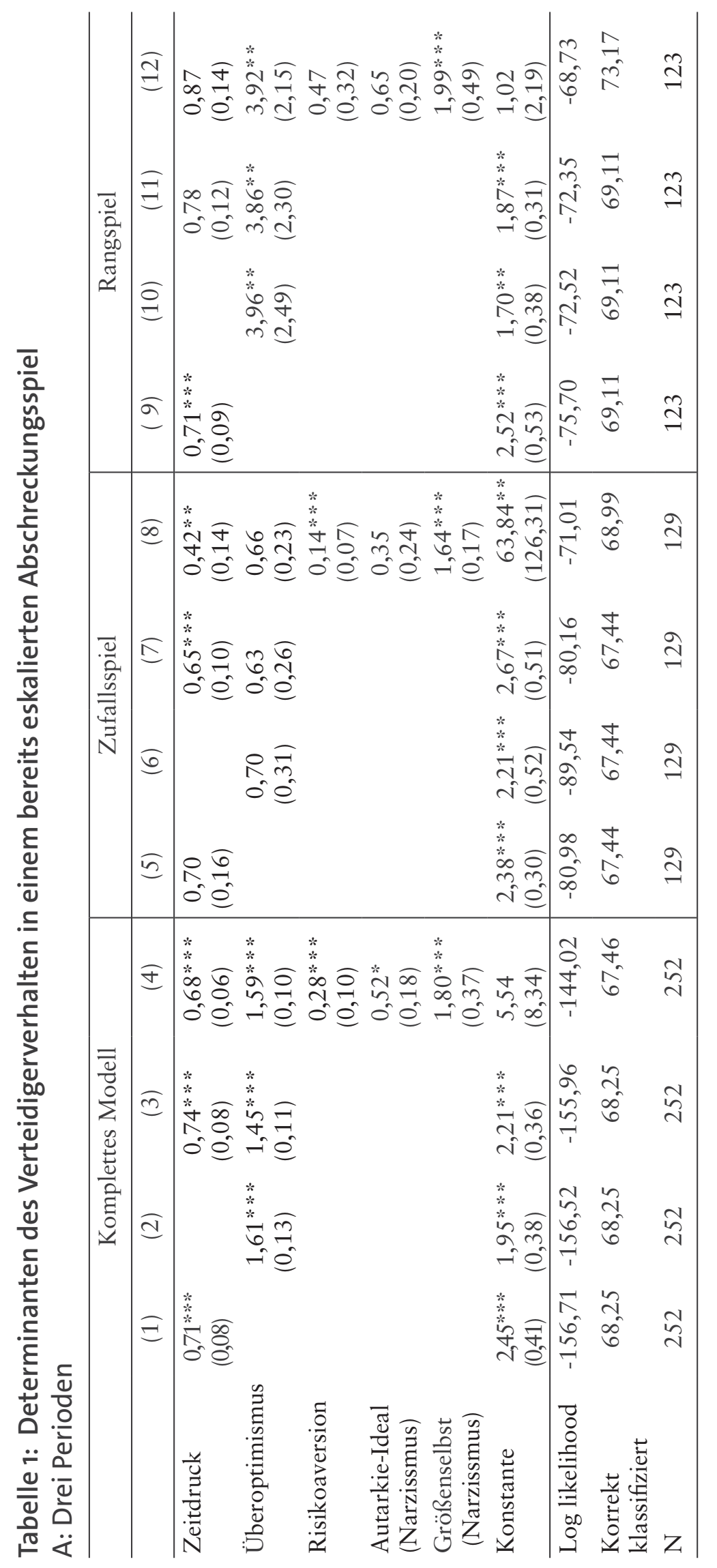




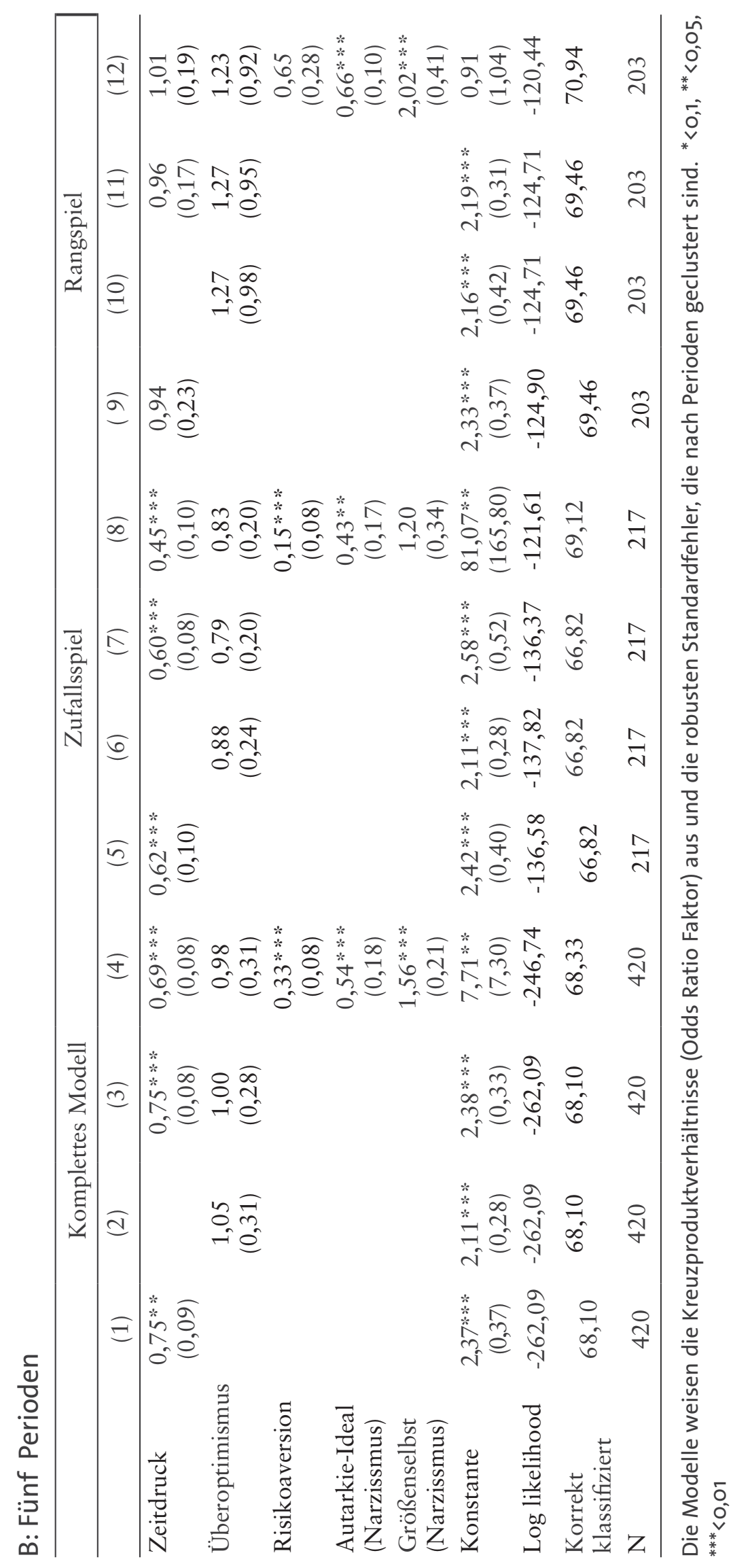


Die empirischen Ergebnisse unterstützen die aus der Literatur abgeleiteten Hypothesen mehrheitlich. Im Widerspruch zu einer zentralen These der GroupthinkLiteratur und zur ersten Hypothese steht das Ergebnis, dass Entscheidungsträger unter Zeitdruck eher einer Eskalation zuneigen. In einem ähnlich gehaltenen Experiment konnten wir auch zeigen, dass bei einem analogen Zeitdruck-Treatment die Entscheidungsqualität abnimmt (Blendin u. Schneider 2012). Zeitdruck scheint Akteure aber im konkreten Abschreckungskontext bereiter zu Konzessionen gegenüber einer monetären Forderung zu machen. In der Überprüfung mit fünf Perioden zeigt sich auch, dass dieser Effekt wie vermutet stärker in der Konstellation eines Zufallsspiels wirkt im Vergleich zu einer Situation, in der sich die Verteidiger angesichts von Informationen über ihr Abschneiden in einem IQ-Test größere Erfolgsaussichten ausmalen können, wenn sie dem Angreifer widerstehen. Dieser Effekt besteht nicht im Falle, wenn die Tests nur die ersten drei Spielperioden erfassen. Allerdings unterscheiden sich die Kreuzproduktverhältnisse nicht substantiell.

Für die zweite Hypothese finden wir vor allem in Tabelle 1-A Unterstützung. So zeigt es sich für alle Fälle (komplettes Modell) wie auch für das Rangspiel, dass überoptimistische Probanden eher zur Konflikteskalation neigen. Die Resultate belegen damit vor allem auch, dass die Selbstüberschätzung besonders dann die Konfliktneigung fördert, wenn die Individuen in einer Situation sind, in der ein Erfolg bei konfliktivem Verhalten von den eigenen Fähigkeiten abzuhängen scheint. Wie für Top-Manager gezeigt werden konnte, erhöht Selbstüberschätzung in einem geeigneten Umfeld das Risikoverhalten (Chatterjee u. Hambrick 2011). Dass die Überoptimismus-These nicht auf alle fünf Perioden bezogen greift, kann mit dem angesprochenen Endrundenverhalten und den Ermüdungseffekten zusammenhängen.

Hypothese 3 ließ uns unabhängig von Entscheidungskontext für narzisstische Entscheidungsträger eine erhöhte Eskalationsneigung vermuten. Dies können wir mit Ausnahme einer Modellkonstellation (Zufallsspiel mit fünf erfassten Perioden) für eine der beiden verwendeten Skalen bestätigen: je höher der Wert auf der Größenselbstskala, desto eher sind Probanden bereit, den Konflikt eskalieren zu lassen. Dieser Zusammenhang ist umso bedenkenswerter, als die in Krisenstäben entscheidenden Politiker wohl ebenso narzisstische Züge aufweisen. So finden Watts et al. (2013) Belege dafür, dass die 42 von ihnen erfassten amerikanischen Präsidenten in Bezug auf das Größenselbst narzisstischer als die Gesamtbevölkerung sind und dass die Tendenz zum Narzissmus im Laufe der Zeit zugenommen hat. Die zweite Dimension des Narzissmus, die das mögliche Autarkie-Ideal der Probanden erfasst, dämpft eher die Risikoneigung. Dies bestätigt, dass die Skalen unterschiedliche Dimensionen des Narzissmus erfassen. Für die weitere Forschung bedeutet dies, dass eine Fokussierung auf das Phänomen des Größenselbst besonders fruchtbar scheint.

Schließlich bekräftigen die logistischen Regressionen, dass risikoscheue Entscheidungsträger tatsächlich eher eine Forderung akzeptieren als risikofreudige Individuen. Im Sinne der vierten Hypothese gilt dieser Zusammenhang besonders für das Zufallsspiel. Die Unterscheidung in das Zufalls- und das Rangspiel unterstreicht, dass Individuen die Anreize in ihr Kalkül einbeziehen, wenn sie sich Ge- 
danken über die Minimierung eines Verlustes machen müssen, wie es für eine Situation der akuten Abschreckung typisch ist.

\section{Schlussfolgerungen}

Die rationale Abschreckungstheorie, wie sie Schelling und andere Militärstrategen begründet haben, ist zentraler Baustein der Sicherheitspolitik der NATO mindestens seit der Ausformulierung der Strategie der flexiblen Antwort. Der Versuch, zwischenstaatliche militärische Krisen, auch zwischen Nuklearmächten, durch dosierte Drohungen kalkulierbar zu machen, gleicht der Brinkmanship-Strategie Schellings, die paradoxerweise auf die Schaffung eines Kriegsrisikos als glaubwürdige Antwort auf eine Sicherheitsbedrohung setzt. Dieser Artikel hat die Kritik an diesem Ansatz aufgegriffen und aufgrund von Entwicklungen in der Sozialpsychologie und der Verhaltensökonomie den Einfluss von Persönlichkeitsmerkmalen und der Entscheidungssituation auf das Verteidigerverhalten in einem spieltheoretischen Abschreckungsszenario untersucht.

Das Abschreckungsexperiment, das wir durchführten, lieferte eindeutige Belege für die These, dass extrarationale Faktoren wie Selbstüberschätzung in der Kombination mit Groupthink-ähnlichen Anreizstrukturen die Eskalationsbereitschaft der Akteure fördern. Damit ergeben sich Übereinstimmungen zu einem Gruppenexperiment, in dem Selbstüberschätzung ebenfalls zum suboptimalen Verhalten der Probanden beitrug (Baker et al. 2013). Als ergebnisrelevant haben sich neben den situativen Bedingungen und den Persönlichkeitsmerkmalen der Akteure Framingeffekte erwiesen. Auch wenn die externe Validität der Ergebnisse aufgrund der abstrakten Entscheidungssituation im Labor zunächst problematisch erscheint, kann davon ausgegangen werden, dass einige der hier diskutierten Faktoren wie Zeitdruck oder Überoptimismus Entscheidungssituationen beeinflussen können. Militärische Entscheidungsträger sollten zwar prinzipiell so geschult werden, dass sie besser mit Zeitdruck als der Durchschnittsproband umgehen können. Aber damit ist die Gefahr einer unnötigen Konflikteskalation nicht gebannt, da die Mitglieder von Krisenstäben oft eine ähnlich problematische Persönlichkeitsstruktur aufweisen wie Topentscheider in der Wirtschaft.

Grundsätzlich scheint es uns möglich, das Problem mangelnder externer Validität durch vergleichende Studien zu lindern. Diese könnten etwa das Entscheidungsverhalten bei Geiselnahmen erfassen, in denen Krisenstäbe im Sinne der Abschreckungstheorie erwägen müssen, ob sie auf die Forderungen eingehen oder ob sie den Konflikt eskalieren lassen. Solche Studien fehlen in der Politikwissenschaft besonders im deutschsprachigen Raum, obwohl es gerade in der Geschichte der Bundesrepublik aufgrund des Terrorismus der Roten Armee Fraktion eigentlich genügend Krisenfälle gab, die sich für einen systematischen Vergleich eignen. Allerdings besteht bei solchen möglichen zeitgeschichtlichen Erkundungen das Problem, dass sich die psychologischen Wesensmerkmale der Entscheidungsträger bestenfalls post hoc erfassen lassen.

Trotz dieser zwangsläufigen Limiten unserer Untersuchungsanlage erachten wir es als ermutigend, dass in vergleichenden Tests zum rationalen Abschreckungsmodell, bei denen reale zwischenstaatliche Krisen untersucht werden, viele theoreti- 
sche Implikationen des Gedankengebäudes empirische Unterstützung erhalten (Fearon 2003). Angesichts der Bedeutung der Abschreckungstheorie in der politischen Praxis genügen die üblichen Evaluationsmaßstäbe jedoch nicht, um die Erklärungskraft der Theorie zu überprüfen. So wirft eine durch extra-rationale Faktoren bereits geringfügig erhöhte Eskalationsbereitschaft die Frage auf, ob die Theorie nicht auch jene Faktoren miteinschließen sollte, die für diese Eskalationstendenzen verantwortlich sind. Obgleich wir keine ausgearbeitete Alternative zur rationalistischen Abschreckungstheorie offerieren können, halten wir es für angebracht, dass Verhaltensbesonderheiten wie etwa der Zusammenhang zwischen Selbstüberschätzung und dem Risikoverhalten in die Theorie eingebaut werden. Dies erfordert zum einen, dass die Modelle auch stärker für risikofreundliche Akteure durchdacht werden und so die dogmatische Annahme der Risikoaversion durchbrochen wird. Für Krisenentscheidungsstäbe ist zu berücksichtigen, dass neben dem Gruppendenken auch die Rekrutierung von narzisstischen oder zur Selbstüberschätzung neigenden Entscheidungsträgern das Risiko einer weiteren Eskalation fördern könnte.

\section{Literatur}

Achen, Christopher H. und Duncan Snidal. 1989. Rational Deterrence Theory and Comparative Case Studies. World Politics 41: 143-169.

Ackerman, Robert A., Edward A. Witt, M. Brent Donnellan, Kali H. Trzesniewski, Richard W. Robins und Deborah A. Kashy. 2011. What Does the Narcissistic Personality Inventory Really Measure? Assessment 18: 67-87

Alicke, Mark D., M. L. Klotz, David L. Breitenbecher, Tricia J. Yurak, und Debbie S. Vredenburg. 1995. Personal Contact, Individuation, and the Better-than-Average Effect Journal of Personality and Social Psychology 68: 804-825.

Baker, Christopher, Blendin, Hanja; und Schneider, Gerald. 2013. Groupthink: Theory and Evidence. Unveröffentlichtes Arbeitspapier, Universität Konstanz.

Ben Zur, Hasida und Shlomo J. Breznitz. 1981. The Effect of Time Pressure on Risky Choice Behavior. Acta Psychologica 47: 89-104.

Blendin, Hanja und Gerald Schneider. 2012. Nicht jede Form von Stress mindert die Entscheidungsqualität: Ein Laborexperiment zur Groupthink-Theorie. Jabrbuch für Handlung- und Entscheidungstheorie 7: 61-80

Brecher, Michael (mit Benjamin Geist). 1980. Decision in Crisis. Israel, 1967 and 1973. Berkeley: University of California Press.

Carnevale, Peter J. D. und Edward J. Lawler. 1986. Time Pressure and the Development of Integrative Agreements in Bilateral Negotiations, Journal of Conflict Resolution 30: 636-659.

Carnevale, Peter J. und Dean G. Pruitt. 1992. Negotiation and Mediation, Annual Review of Psychology 43: 531-582.

Carnevale, Peter J., Kathleen M. O’Connor und Christopher McCusker. 1993. Time Pressure in Negotiation and Mediation. In Time Pressure and Stress in Human Judgement and Decision Making, Hrsg. Ola Svenson, O. und A. John Maule, 117-127. New York: Plenum Press. 
Chatterjee, Arijit und Donald C. Hambrick 2011. Executive Personality, Capability Cues, and Risk Taking: How Narcissistic CEOs React to their Successes and Stumbles. Administrative Science Quarterly 56: 202-237.

Coetzee, J. M. 2008. Diary of a Bad Year. London: Penguin.

Davis, James W. Hrsg. 2013. Psychology, Strategy and Conflict. Perceptions of Insecurity in International Relations. London and New York: Routledge.

De Dreu, Carsten K. W. 2003. Time Pressure and Closing of the Mind in Negotiation, Organizational Behavior and Human Decision Processes 91: 280-295.

Denecke, Friedrich-Wilhelm und Hurkard Hilgenstock. 1989. Narzissmus Inventar (NI), Bern: Hans-Huber-Verlag.

Eiser, J. Richard, Sabine Pahl und Yvonne R. A. Prins. 2001. Optimism, Pessimism, and the Direction of Self-Other Comparisons. Journal of Experimental Social Psychology 37: 77-84.

Farnham, Barbara. 1992. Roosevelt and the Munich Crisis: Insights from Prospect Theory. Political Psychology 13: 205-235.

Farnham, Barbara. 1994. Avoiding Losses/Taking Risks: Prospect Theory and International Conflict. Ann Arbor: University of Michigan Press.

Fearon, James D. 1994. Domestic Political Audiences and the Escalation of International Disputes. American Political Science Review 88: 577-592.

Fearon, James D., 1995. Rationalist Explanations for War, International Organization 49: 379-414.

Fearon, James D. 2003. Selection Effects and Deterrence. International Interactions 28: 5-29.

Fischbacher, Urs. 2007. Z-Tree: Zurich Toolbox for Ready-Made Economic Experiments. Experimental Economics 10: 171-178

Frei, Daniel 1982. Risks of Unintentional Nuclear War. Totowa, N.J. : Allanheld.

Gabriel, Marsha. T., Joseph W. Critelli und Juliana S. Ee. 1994. Narcissistic illusions in self-evaluations of intelligence and attractiveness. Journal of Personality 62: 143-155.

Gigerenzer, Gerd, Ulrich Hoffrage und Heinz Kleinbölting. 1991. Probabilistic Mental Models: A Brunswikian Theory of Confidence. Psychological Review 98: 506-528.

Green, Philip. 1966. Deadly Logic: The Theory of Nuclear Deterrence. Columbus: Ohio State University Press.

Greiner, Ben. 2004. An Online Recruitment System for Economic Experiments. Forschung und wissenschaftliches Rechnen 2003. GWDG Bericht 63: 79-93

Halperin, Morton. 1987. Nuclear Fallacy: Dispelling the Myth of Nuclear Strategy. Cambridge: Ballinger.

Hermann, Margaret G. 1979. Indicators of Stress in Policymakers during Foreign Policy Crises. Political Psychology 1: 27-46.

Holt, Charles A. und Susan K. Laury. 2002. Risk Aversion and Incentive Effects. American Economic Review 92: 1644-1655.

Ibañez, Marcela, Simon Czermak und Matthias Sutter. 2009. Searching for a Better Deal On the Influence of Group Decision Making, Time Pressure and Gender on Search Behavior. Journal of Economic Psychology 30: 1-10.

Janis, Irving L. 1971. Groupthink among Policy Makers. In Sanctions for Evil: Sources of Social Destructiveness. Hrsg. Comstock Nevitt und Craig Sanford, 71-89. San Francisco: Jossey-Bass. 
Irving Janis 1. 1972. Victims of Groupthink: A Psychological Study of Foreign-Policy Decisions and Fiascoes. Boston: Houghton Mifflin.

Janis, Irving L. und Leon Mann, L. 1977 Decision Making. A Psychological Analysis of Conflict, Choice, and Commitment, New York: Macmillan.

Jervis, Robert. 1976. Perception and Misperception in International Politics. Princeton: Princeton University Press.

Jervis, Robert. 1979. Review: Deterrence Theory Revisited. World Politics 31: 289-324.

Jervis, Robert. 1988. War and Misperception. Journal of Interdisciplinary History 18: 675-700.

Johnson, Dominic D. P. 2004, Overconfidence and War. The Havoc and Glory of Positive Illusions, Cambridge: Harvard University Press.

Johnson, Dominic D. P., Rose McDermott, Emily S. Barrett, Jonathan Cowden, Richard Wrangham, Matthew H. McIntyre und Stephen Peter Rosen. 2006. Overconfidence in Wargames: Experimental Evidence on Expectations, Aggression, Gender and Testosterone. Proceedings of the Royal Society B: Biological Sciences 273: 2513-2520.

Johnson, Dominic D. P. und James H. Fowler. 2011. The Evolution of Overconfidence. Nature 477: 317-320.

Johnson, Dominic D.P., Rose McDermott, Jon Cowden und Dustin Tingley 2012. Dead Certain: Confidence and Conservatism Predict Aggression in Simulated International Crisis Decision-Making. Human Nature 23: 98-126.

Jones, Daniel N. und Delroy L. Paulhus 2010. Different provocations trigger aggression in narcissists and psychopaths. Social Psychology and Personality Bulletin 1: 12-18.

Kahneman, Daniel und Jonathan Renshon. 2007. Why Hawks Win. Foreign Policy 158: 34-38.

Kahneman, Daniel und Amos Tversky. 1979. Prospect Theory: An Analysis of Decision under Risk. Econometrica 47: 263-292

Keren, Gideon. 1991. Calibration and Probability Judgements: Conceptual and Methodological Issues. Acta Psychologica 77: 217-273.

Kochan, Thomas A. und Todd Jick. 1978. The Public Sector Mediation Process: A Theory and Empirical Examination. Journal of Conflict Resolution 22: 209-240.

Krämer, Ulrike Sabrina und Gerald Schneider. 2003. Faire Formeln. Psychologische und prozedurale Einflussfaktoren auf die Lösung von distributiven Konflikten.. Kölner Zeitschrift für Soziologie und Sozialpsychologie 55: 55-78.

Langer, Ellen J. 1975. The Illusion of Control. Journal of Personality and Social Psychology 32: 311-328.

Lebow, Richard Ned und Janice Gross Stein. 1989. Rational Deterrence Theory: I Think, Therefore I Deter. World Politics 41: 208-224.

Levy, Jack S. 1992a. An Introduction to Prospect Theory. Political Psychology 13: 171-86.

Levy, Jack S. 1992b. Prospect Theory and International Relations: Theoretical Applications and Analytical Problems. Political Psychology 13: 283-310.

McKenna, Frank P. 1993. It Won't Happen to me: Unrealistic Optimism or Illusion of Control. British Journal of Psychology 84: 39-50.

Mercer, Jonathon. 2012. Audience costs are toys. Security Studies 21: 398-404.

Mercer, Jonathan. 2010. Emotional beliefs. International Organization 64: 1-31. 
Mosterd, Igor und Rutte, Christel G. 2000. Effects of Time Pressure and Accountability to Constituents on Negotiation, International Journal of Conflict Management 11: 227247.

O’Neill, Barry. 1992. Deterrence Theory Discussion: II: Are Game Models of Deterrence Biased towards Arms-Building? Wagner on Rationality and Misperception. Journal of Theoretical Politics 4: 459-477.

O’Neill, Barry. 2002. Risk aversion in international relations theory. International Studies Quarterly 454: 617-40

Pailing, Andrea, Julian Boon und Vincent Egan. 2014. Personality, the Dark Triad and Violence. Personality and Individual Differences 67: 81-86.

Pallier, Gerry, Rebecca Wilkinson, Vanessa Danthiir, Sabina Kleitman,

Goran Knezevic, Lazar Stankov und Richard D. Roberts .2002. The Role of Individual Differences in the Accuracy of Confidence Judgments. Journal of General Psychology 129: 257-299.

Pincus, Aaron L. und Lukowitsky, Mark R. 2010. Pathological narcissism and narcissistic Personality disorder. Annual Review of Clinical Psychology 6: 421-446.

Pinfari, Marco 2011. Time to Agree: Are Deadlines Good for Peace Negotiations? Journal of Conflict Resolution 55: 683-709.

Powell, Robert. 1987. Crisis Bargaining, Escalation, and MAD, American Political Science Review 81: 717-735.

Rapoport, Anatol 1964. Strategy and Concsience. New York: Harper\&Row.

Rapoport, Anatol 1992. Comments on "Rationality and Misperceptions in Deterrence Theory". Journal of Theoretical Politics 4: 479 - 484.

Raskin, Robert und Calvin S. Hall 1981. The Narcissistic Personality Inventory: Alternative Form Reliability and Further Evidence of Construct Validity, Journal of Personality Assessment 45: 159-162.

Resick, Christian J., Daniel S. Whitman, Steven M. Weingarden und Nathan J. Hiller 2009. The Bright-Side and the Dark-Side of CEO Personality: Examining Core Self-Evaluations, Narcissism, Transformational Leadership, and Strategic Influence. Journal of Applied Psychology 94: 1365-1381.

Russo, J. Edward und Schoemaker, Paul J. H. 1992. Managing Overconfidence, Sloan Management Review. Reprint Series 33: 6-17.

Schelling, Thomas C. 1958. The Reciprocal Fear of Surprise Attack. The RAND Corporation.

Schelling, Thomas C. 1960. The Strategy of Conflict, Harvard: University Press.

Schelling, Thomas C. 1966. Arms and Influence, Yale: University Press.

Schelling, Thomas C. 2006. An Astonishing Sixty Years. The Legacy of Hiroshima. PNAS 103: 6089-6093.

Schneider, Gerald, Krämer, Ulrike Sabrina. 2004. The Limitations of Fair Division: An Experimental Assessment of Three Procedures. Journal of Conflict Resolution 48: 506524.

Schütz, A., Marcus, B. und Sellin, I. 2004. Die Messung von Narzissmus als Persönlichkeitskonstrukt: Psychometrische Eigenschaft einer Lang- und einer Kurzform des Deutschen NPI (Narcissistic Personality Inventory), Diagnostica 50: 202-218.

Snyder, Jack, and Borghard, Erica. 2011. The Cost of Empty Threats: A Penny, Not a Pound. American Political Science Review 105: 437-56. 
Stein, Janice. G. 1988. Building Politics into Psychology: The Misperception of Threat, Political Psychology 9: 245-271.

Sutter, Mathias, Martin Kocher und Sabine Strauß. 2003. Bargaining Under Time Pressure in an Experimental Ultimatum Game, Economics Letters 81: 341-347.

Svenson, Ola. 1981. Are We All Less Risky and More Skillful Than Our Fellow Drivers?, Acta Psychologica 47: 143-148.

Svenson, Ola und A. John Maule (Hrsg.) 1993. Time Pressure and Stress in Human Judgment and Decision Making, New York: Plenum Press.

Thaler, Richard H. , Amos Tversky, Daniel Kahneman und Alan Schwartz. 1997. The Effect of Myopia and Loss Aversion on Risk Taking: An Experimental Test. Quarterly Journal of Economics 112: 647-661.

Tomz, Michael. 2007. Domestic Audience Costs in International Relations: An Experimental Approach. International Organization 61: 821-40.

Trachtenberg, Marc. 2012. Audience Costs: An Historical Analysis. Security Studies 21: $3-42$.

Wagner, R. Harrison 1992. Rationality and Misperception in Deterrence Theory, Journal of Theoretical Politics 4: 115-141.

Watts, Ashley L., Scott O. Lilienfeld, Sarah Francis Smith, Joshua D. Miller, W. Keith Campbell, Irwin D. Waldman, Steven J. Rubenzer und Thomas J. Faschingbauer 2013. The Double-Edged Sword of Grandiose Narcissism: Implications for Successful and Unsuccessful Leadership Among U.S. Presidents. Psychological Science 24: 2379-2389.

Weinstein, Neil D. 1980. Unrealistic Optimism About Future Life Events, Journal of Personality and Social Psychology 39: 806-820.

Zagare, Frank C. 1996. Classical Deterrence Theory: A Critical Assessment, International Interactions 21: 365-387.

Zagare, Frank. C. und Mark D. Kilgour. 2000. Perfect Deterrence, Cambridge: Cambridge University Press.

Zagare, Frank C. 2004. Reconciling Rationality with Deterrence: A Re-Examination of the Logical Foundations of Deterrence Theory. Journal of Theoretical Politics 16: 107141.

Zeigler-Hill, Virgil, Brian Enjaian und Lauren Essa 2013. The Role of Narcissistic Personality Features in Sexual Aggression. Journal of Social and Clinical Psychology. 32:. 186-199.

Zook, Darren C. 2000. A Culture of Deterrence: Nuclear Myths and Cultural Chauvinism in South Asia. World Policy Journal 17: S. 39-46.

Zakay, Dan und Joseph Glicksohn. 1992. Overconfidence in a Multiple-Choice Test and ist Relationship to Achievement. The Psychological Record. 42: 519-524 